Canadian

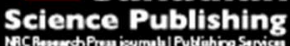

Applied Physiology, Nutrition, and Metabolism Physiologie appliquée, nutrition et métabolisme

\title{
Role of vitamin A oral supplementation on oxidative stress and inflammatory response in the liver of trained rats
}

\begin{tabular}{|r|l|}
\hline Journal: & Applied Physiology, Nutrition, and Metabolism \\
\hline Manuscript ID & apnm-2017-0193.R2 \\
\hline Manuscript Type: & Article \\
\hline Complete List of Authors: & $\begin{array}{l}\text { Petiz, Lyvia; Universidade Federal do Rio Grande do Sul, Bioquímica } \\
\text { Kunzler, Alice; Universidade Federal do Rio Grande do Sul, Bioquímica } \\
\text { Bortolin, Rafael; Universidade Federal do Rio Grande do Sul, } \\
\text { Gasparotto, Juciano; Universidade Federal do Rio Grande do } \\
\text { Sul, biochemistry } \\
\text { Matté, Cristiane; Universidade Federal do Rio Grande do Sul, Biochemistry } \\
\text { Moreira, José Claudio; UFRGS } \\
\text { Gelain, Daniel; Universidade Federal do Rio Grande do Sul, }\end{array}$ \\
\hline Is the invited manuscript for \\
consideration in a Special \\
Issue? :
\end{tabular}

\section{SCHOLARONE"}

Manuscripts 


\section{Role of vitamin A oral supplementation on oxidative stress and inflammatory response in the liver of trained rats}

Authors: Lyvia Lintzmaier Petiz ${ }^{1}$, Alice Kunzler ${ }^{1}$, Rafael Calixto Bortolin ${ }^{1}$, Juciano Gasparotto $^{1}$, Cristiane Matté ${ }^{1}$, José Claudio Fonseca Moreira ${ }^{1}$, Daniel Pens Gelain ${ }^{1}$ Corresponding Author: Lyvia Lintzmaier Petiz, Rua Ramiro Barcelos, 2600 - Instituto de Ciências Básicas da Saúde, prédio anexo - CEP 90035-003 - Porto Alegre, RS, Brazil.

Phone number: +55 5133085577 Fax number: +55 5133085540

E-mail: 1yviapetiz@gmail.com

Affiliation: ${ }^{1}$ Departamento de Bioquímica, Instituto de Ciências Básicas da Saúde, Universidade Federal do Rio Grande do Sul, Rua Ramiro Barcelos, 2600 - prédio anexo - CEP 90035-003 - Porto Alegre, RS, Brazil.

Porto Alegre, RS, Brazil.

Lyvia L. Petiz - lyviapetiz@gmail.com (corresponding author)

Alice Kunzler - alice.bio@hotmail.com

Rafael Calixto Bortolin - rafaelbortolin@hotmail.com

Juciano Gasparotto - juciano.gasparotto@gmail.com

Cristiane Matté - cristianematte@gmail.com

José Claudio Fonseca Moreira - jcfm@ufrgs.br

Daniel Pens Gelain - dgelain@yahoo.com.br 


\section{ABSTRACT}

The use of dietary supplements to enhance the benefit of exercise training is a common practice. The liver is the organ where all substances are metabolized, and certain supplements have been associated with liver injury. Vitamin A (VA), a liposoluble vitamin stored in the liver, is commonly used as an antioxidant supplement. Here, we evaluated the effect of chronic VA supplementation on oxidative damage and stress parameters in trained rats. Animals were divided into the following groups: sedentary (SE), sedentary/VA (SE+VA), exercise training (ET), and exercise training/VA $(\mathrm{ET}+\mathrm{VA})$. During 8 weeks, animals were subjected to swimming $(0,2,4,6 \%$ body weight) 5 days/week and a VA daily intake of 450 retinol equivalents/day. Parameters were evaluated by enzymatic activity analysis, ELISA, and western blotting. VA caused liver lipid peroxidation and protein damage in exercised rats and inhibited the increase in HSP70 expression acquired with exercise alone. ET group showed higher levels of antioxidant enzyme activity, and VA inhibited this adaptation. Expression of the proinflammatory cytokines, interleukin (IL)- $1 \beta$ and tumor necrosis factor- $\alpha$, was reduced in the ET+VA group, while the anti-inflammatory cytokine, IL-10, was increased. Western blotting showed that both exercised groups had lower levels of the receptor for advanced glycation end products (RAGE), suggesting that VA did not affect this receptor. Our study demonstrated that, although VA caused oxidative damage, a controlled administration might exert anti-inflammatory effects. Further studies with higher VA doses and longer ET interventions would elucidate more the effects of the supplementation and exercise on liver parameters.

Keywords: antioxidant enzymes; exercise; cytokines; liver; reactive oxygen species; vitamin A; western blotting; Wistar rat model 


\section{Introduction}

Regular exercise training (ET) is recommended for the prevention and treatment of several disorders (Neufer et al. 2015), in addition to maintain an anti-inflammatory state, characterized by small adipocyte size and the presence of anti-inflammatory cell types such as regulatory $\mathrm{T}$ cells and M2-type macrophages (Gleeson et al. 2011). Paradoxically, it is also clear that the large increase in oxygen consumption, that can reach up to 10-15-fold during ET (Sen 1995), leads to the production of reactive oxygen species (ROS), high concentrations of which can cause oxidative stress and tissue damage (Chance et al. 1979). Oxidative stress is characterized by the disruption of redox signaling control within the cell (Jones 2006), which can have an impact on several cellular functions such as cell differentiation, proliferation, migration, quiescence, and death (Sarsour et al. 2009; Kunzler et al. 2016). An imbalance in ROS production occurs when the formation of ROS is not followed by a proper antioxidant response of the cell, and it can be caused by an increase or decrease in ROS production combined with a wide range of alterations in the activation and expression of antioxidant enzymes and/or the presence of non-enzymatic antioxidants (Banerjee et al. 2003).

The liver is one of the most metabolically challenged organs during ET, mainly owing to its key role in the removal of lactate from circulation for gluconeogenesis and maintenance of blood glucose levels (Brooks 1986; Coker and Kjaer 2005). The liver is a major regulator of energy metabolism at a systemic level, and maintaining its function is crucial to sustain the performance of other organs and tissues during ET. Acute or chronic exercise can affect liver function. An acute bout of exercise can increase hepatic protein synthesis without changes in fat content, reduce hepatic blood flow, and cause a significant imbalance in ROS production (Shephard and Johnson 2015). Furthermore, in 
streptozotocin-induced diabetic rats, chronic ET has been shown to prevent the impairment of hepatic redox defenses, such as decreased expression of liver antioxidant enzymes and increased ROS levels (Lima et al. 2015). Although consumption of a weight-loss diet is still the most effective treatment for non-alcoholic fatty liver disease, ET alone has been shown to be highly beneficial in treating this disease. ET reduces insulin resistance and increases the expression of genes responsible for fatty acid metabolism in this condition (Oh et al. 2014; Ordonez et al. 2015). The redox imbalance caused by exercise may lead to inflammation, wherein increased ROS levels are related to inflammatory processes (Kosmidou et al. 2002). Regular exercise can decrease the levels of inflammatory cytokines, adipokines, and other injury-related markers in the liver (Gleeson 2007). These anti-inflammatory effects appear to occur due to three main factors: reduced visceral fat, increased production and release of anti-inflammatory cytokines such as interleukin (IL)-10, and decreased expression of toll-like receptors in immune cells (Gleeson et al. 2011).

Dietary supplementation during ET is a common practice to enhance performance or to prevent/treat diseases. However, the effects of different combinations of dietary supplements and regular ET are unknown. Several natural supplements have been associated with liver injury, such as green tea extract and other herbal preparations, usnic acid, and vitamin A (VA) (Garcia-Cortes et al. 2016). VA is a fatsoluble vitamin essential for many key biological processes, including vision, embryonic development, gene transcription, and immune responses (Chapman 2012). It can be found in the form of all-trans retinol and retinyl esters (in foods from animal sources) and in the form of pro-vitamin A carotenoids, such as $\beta$-carotene (in foods from vegetable sources) (Blomhoff and Blomhoff 2006). The liver is one of the main sites of VA metabolism and storage, and both the hepatic cells, parenchymal 
(hepatocytes) and stellate cells, participate in these processes (D'Ambrosio et al. 2011). Oral intake or administration of therapeutic doses of VA may induce hepatotoxicity through hypervitaminosis (Geubel et al. 1991). Furthermore, studies evaluating the effect of VA supplementation over oxidative damage induced by different stressors in the liver demonstrated that VA may act as an antioxidant molecule (protective), but also as pro-oxidant (leading to liver damage), and therefore the effects of VA will largely depend on the dose, mechanism of administration and the characteristics of the stressors (de Oliveira et al. 2009; Schnorr et al. 2011; Wang et al. 2014; Shimizu et al. 2017).

Thus, considering the major role of liver in VA metabolism and storage, and the wide range of effects of VA on liver oxidative stress regulation, the present work was designed to evaluate the effect of VA supplementation on parameters of ROS-mediated damage, antioxidant defense, cell stress, and pro-inflammatory modulation in the liver of rats submitted to intense swimming ET. In previous works, we observed that VA supplementation with ET impaired some exercise-acquired benefits in highly demanded tissues of trained rats, by decreasing the activity of antioxidant defenses and causing lipoperoxidation in the lungs (Gasparotto et al. 2015) and skeletal muscle (Petiz et al. 2017). Due to the role of the liver in the regulation of energetic homeostasis during exercise and also in the metabolism of VA, this study is intended to improve the understanding of the mechanisms by which VA supplementation presented potentially harmful effects when combined with ET.

\section{Materials and Methods}

\section{Ethics}


All experimental assays in this work were conducted in accordance with the National Institute of Health Guide for the Care and Use of Laboratory Animals (2011) and followed the guidelines of the Brazilian Society of Animal Science Experimentation (SBCAL). Before beginning the research project, it was approved by the Ethical Committee for Animal Experimentation of the Federal University of Rio Grande do Sul (CEUA-UFRGS) under the accession number 25837.

\section{Animals}

Male Wistar rats (7-week-old; weight: 250-300 g) were obtained from our own breeding colony at the Federal University of Rio Grande do Sul and kept in plastic cages, with a maximum of 4 animals per cage. Animals were maintained in a room with an ambient temperature of $23 \pm 1^{\circ} \mathrm{C}$ and $12 \mathrm{~h}$ light-dark cycle (7am-7pm), with $\mathrm{ad}$ libitum access to food and water.

\section{Study design}

Animals were randomized into four groups: sedentary (SE), sedentary supplemented with VA (SE+VA), exercise training (ET), and exercise training supplemented with VA (ET+VA). Throughout the 8 weeks, the exercised and sedentary groups were administered 450 retinol equivalents (RE) (1500 IU)/kg/day of retinyl palmitate (Arovit ${ }^{\circledR}$; Bayer, Rio de Janeiro, RJ, Brazil) or its vehicle (saline) daily by intragastric gavage. The chosen dose of $450 \mathrm{RE}$ was calculated based on the human equivalent dose

(HED), using the dose-by-factor approach (Nair et al. 2016). The exercised groups were subjected to a protocol of 60 min of swimming 5 days/week, while the sedentary groups 
remained in shallow water for $20 \mathrm{~min} 5$ days/week. ET and supplementation protocols were performed between 6-8 pm.

\section{Exercise training protocol}

The training protocol lasted 8 weeks in total. It was conducted between 6 and 8 pm, in a swimming tank for rodents with water at $31 \pm 1{ }^{\circ} \mathrm{C}$. During the first week, all animals remained in shallow water for 20-60 min each day. For the next 2 weeks, training started with $10 \mathrm{~min} /$ day and progressed to $60 \mathrm{~min} /$ day. Over the following 5 weeks, training consisted of $60 \mathrm{~min} /$ day, 5 days/week, with an overload attached to the animal's torso, progressing each week ( $0,2,4,6 \%$ body weight) (Gobatto et al. 2001). Animals were weighed once a week and the values were utilized to calculate the overload. After each session, animals were towel-dried and returned to their cages.

\section{Tissue sampling}

After a $24 \mathrm{~h}$ interval from the last exercise bout and VA supplementation, animals were euthanized by decapitation, and their blood and liver tissue samples were collected. Blood samples were centrifuged at $1500 \times g$ for $10 \mathrm{~min}$ for serum isolation. Tissue samples were homogenized in phosphate buffer $(\mathrm{PB})$ and centrifuged $(3000 \times g, 10$ min), and sample supernatants were used for analysis. Protein content was quantified by the Lowry method (Lowry et al. 1951) using bovine serum albumin as standard. For western blotting, the tissue was homogenized in RIPA buffer (20 mM Tris-HCl pH 7.5; $150 \mathrm{mM} \mathrm{NaCl} ; 1 \mathrm{mM}$ EDTA; 1 mM EGTA; $2.5 \mathrm{mM}$ sodium pyrophosphate; 1\% sodium deoxycholate; $1 \%$ Tergitol-type NP-40; $1 \mathrm{mM} \beta$-glycerophosphate; $1 \mathrm{mM}$ sodium orthovanadate; $1 \mu \mathrm{g} / \mathrm{mL}$ leupeptin) and centrifuged, following which the 
homogenate was added to Laemmli-buffer (62.5 mM Tris-HCl pH 6.8; 1\% SDS; $10 \%$ glycerol) with $10 \% \beta$-mercaptoethanol.

\section{Plasma assays}

\section{Biochemical parameters}

Serum levels of aspartate transaminase (AST) and alanine transaminase (ALT) were evaluated using standard commercially available biological kits following the manufacturer's instructions (Labtest, São Paulo, Brazil).

\section{Liver homogenate assays}

\section{Redox parameters}

Thiobarbituric acid reactive species (TBARS) levels in the liver samples were quantified as an index of lipid peroxidation (Draper and Hadley 1990). First, samples were deproteinized by $10 \%$ trichloroacetic acid (TCA), followed by heating at $100{ }^{\circ} \mathrm{C}$ for 25 min with $0.67 \%$ thiobarbituric acid. TBARS levels were then quantified spectrophotometrically at a wavelength of $532 \mathrm{~nm}$. Oxidative damage to proteins was quantified by detection of carbonyl groups (Levine et al. 1990), which involved incubation of sample proteins, previously precipitated with 20\% TCA, with 2,4dinitrophenylhydrazine (DNPH), followed by spectrophotometric quantification at 370 $\mathrm{nm}$. Nitrotyrosine content was detected by an indirect enzyme-linked immunosorbent assay (ELISA) using a polyclonal antibody (Abcam, Cambridge, UK). Quantification of antioxidant enzyme activity was performed through kinetic spectrophotometric assays. Superoxide dismutase (SOD; EC 1.15.1.1) activity was determined as the inhibition of superoxide anion-dependent adrenaline auto-oxidation in the presence of the liver 
sample at $480 \mathrm{~nm}$ (Misra and Fridovich 1972). Catalase (CAT; EC 1.11.1.6) activity was measured as the decrease in hydrogen peroxide $\left(\mathrm{H}_{2} \mathrm{O}_{2}\right)$ absorbance in the presence of the liver sample at $240 \mathrm{~nm}$ (Aebi 1984).

\section{ELISA}

Indirect ELISA assay was performed using antibodies to detect the pro-inflammatory cytokines [IL-1 $\beta$, tumor necrosis factor- $\alpha$ (TNF- $\alpha)$, and IL-6], the anti-inflammatory cytokine (IL-10), the advanced glycation end products (AGEs), 4-hydroxynonenal (4HNE), and carboxymethyl lysine (all the six antibodies were purchased from Abcam, Cambridge, UK). The liver tissue samples homogenized in PB were placed in a specific round-bottom plate and left overnight for sample adherence. They were then incubated overnight with primary antibody in a 1:1000 dilution range, followed by incubation with secondary antibody for $2 \mathrm{~h}$ in a 1:2000 dilution range. Between every step, the plate was washed three times with $\mathrm{PB}$ in $0.05 \%$ Tween-20, and all incubations were conducted at $4{ }^{\circ} \mathrm{C}$ under constant agitation (45 rpm). Immunoreactivity was detected with a colorimetric assay using the TMB Chromogen solution for ELISA (Thermo Fisher Scientific, Rockford, USA).

\section{Western blotting}

After subjecting the liver samples $(20 \mu \mathrm{g})$ homogenized in RIPA buffer with $10 \% \beta$ mercaptoethanol to sodium dodecyl sulfate-polyacrylamide gel electrophoresis (SDSPAGE), transference was achieved by a semi-dry transfer of proteins to a nitrocellulose membrane (Millipore, Bedford). Protein content was then determined by Ponceau S staining. Membranes containing the sample proteins were washed using Tris-Tween- 
Buffer-Saline (TTBS - $100 \mathrm{mM}$ Tris, $\mathrm{pH} 7.5 ; 0.9 \% \mathrm{NaCl} ; 0.1 \%$ Tween-20) for posterior blocking with 5\% non-fat dry milk for $1 \mathrm{~h}$ (room temperature). After TTBS washes, membranes were incubated with primary antibodies (1:1000 dilution) against HSP70 (Cell Signaling Technology, Beverly, USA) and the receptor for AGEs (RAGE) and $\beta$ actin (Sigma Chemical, St. Louis, USA) for $2 \mathrm{~h}$ at room temperature, followed by incubation with anti-rabbit/mouse horseradish peroxidase-linked secondary antibodies (1:2000 dilution; Cell Signaling Technology, Beverly, USA) for $1 \mathrm{~h}$ at room temperature. Immunoreactivity was detected through chemiluminescence using the SuperSignal West Pico Chemiluminescent kit (Thermo Scientific, Rockford, USA). Densitometry analysis was conducted with ImageJ software, and results were expressed as the ratio of target protein $/ \beta$-actin.

\section{Statistical analysis}

Statistical analysis was performed by analysis of variance (ANOVA) followed by Bonferroni's test for average comparison, using GraphPad Prism version 5.0 (GraphPad Software Inc, San Diego, USA). The data are expressed as mean \pm standard error of mean (SEM) and values were considered significant at $\mathrm{p}<0.05$.

\section{Results}

\section{Serum ALT and AST levels}

ALT and AST are enzymes expressed in the liver, and their serum activities indicate the degree of liver tissue damage. ALT activity (Fig. 1A) was significantly enhanced in both ET and ET+VA groups, compared to that in both the sedentary groups. AST (Fig. 1B) had lower activity in both the exercised groups compared to that in the SE group. 
These results showed that ET and VA supplementation both have an impact on liver function.

\section{ROS-mediated damage and stress parameters}

We analyzed the oxidative damage biomarkers and antioxidant enzyme activities in the liver tissue. The ET+VA group showed increased lipoperoxidation compared to other groups (Fig. 2A). This effect was confirmed by the assessment of levels of 4-HNE, an end product of lipid peroxidation chain reaction (Fig. 2B). Analysis of oxidative protein damage showed decreased carbonyl formation in the SE+VA group compared to that in the SE group; however, carbonyl formation in the other groups was not significantly affected (Fig. 2C). Nitrotyrosine accumulation, on the other hand, was increased in the ET group, and VA supplementation did not change this effect (Fig. 2D). AGE formation, which is increased in several conditions related to oxidative/nitrosative stress and metabolic dysfunction (such as diabetes), was decreased by VA supplementation itself and in ET and ET+VA groups (Fig. 2E). Finally, the levels of the chaperone protein, HSP70, which is induced under conditions of cellular stress, were evaluated by western blotting. ET enhanced HSP70 levels in the liver, but VA supplementation inhibited this effect (Fig. 2F).

\section{Antioxidant enzyme activities}

The activities of the antioxidant enzymes, SOD and CAT, were enhanced in response to activation of endogenous ROS production. SOD (Fig. 3A) and CAT (Fig. 3B) activities were both increased in the ET group; however, VA supplementation inhibited this effect. 


\section{Modulation of inflammatory parameters}

Next, we measured cytokine levels to evaluate the effect of VA supplementation on the pro-inflammatory stimulation caused by ET. IL-1 $\beta$ levels were increased in the ET group, and VA supplementation inhibited this effect (Fig. 4A). Basal levels of liver TNF- $\alpha$ were unaffected in the ET group; however, the ET+VA group presented decreased TNF- $\alpha$ levels compared to all other groups (Fig. 4B). The pro-inflammatory cytokine, IL-6, is also an anti-inflammatory myokine, which is generally stimulated during muscle contraction and ET. Levels of IL-6 in the liver were increased in the SE+VA and ET groups, compared to SE group; however, this effect was inhibited in the ET+VA group (Fig. 4C). The levels of the anti-inflammatory cytokine, IL-10, were enhanced in the ET group as compared with that in the SE group, and the combination of ET and VA further stimulated this effect (Fig. 4D). Finally, since increased RAGE levels have been associated with chronic pro-inflammatory conditions (Wautier et al. 2016), their levels were evaluated. Although RAGE levels were increased by VA supplementation alone, the ET and ET+VA groups demonstrated a significant decrease in RAGE levels compared to the SE and SE+VA groups, respectively (Fig. 4E).

\section{Discussion}

While ET increased ALT and decreased AST levels, VA supplementation did not have an influence on these parameters. ALT and AST are well-known serum markers used to assess and monitor liver damage. In athletes, however, these enzymes may originate from different tissues; while ALT comes mainly from the liver, AST can be linked to 
muscle cell leakage (Banfi et al. 2012). Indeed, increased levels of ALT and AST are a common response to intense ET (Kayatekin et al. 2002; Koury et al. 2016).

It is well established that vigorous exercise increases ROS production and antioxidant activity due to the enhanced oxygen demands of the tissue and considerable increase in blood flow (Finaud et al. 2006). As a consequence, oxidative damage resulting from long-term ET leads to an antioxidant adaptation in tissues such as skeletal muscle, liver and also the DNA (Abruzzo et al. 2013; Radak et al. 2013; Pillon Barcelos et al. 2017). The liver response to exercise may vary, training intensity being the major factor that determines oxidative damage and antioxidant adaptation. VA presents variable redox-active properties in biological systems, and usually, its effects are related to prevention of oxidative damage (Ozhogina and Kasaikina 1995; Powers and Lennon 1999; Schroder et al. 2001). However, as a lipophilic compound, VA can easily interact with membrane lipids, and the conjugated double bonds present in its structure can facilitate the formation of conjugated dienes during lipid peroxidation chain reactions, if concentrations are high (Halliwell 2006). In our study, the overall effect of VA on tissue redox activity and antioxidant defense varied according to the properties of each tissue fraction. Animals subjected to intense swimming ET showed basal levels of lipid peroxidation products (TBARS and 4-HNE) and protein carbonyls, but VA supplementation increased lipid damage. On the other hand, nitrotyrosine formation, a hallmark of peroxynitrite-mediated protein damage, was increased in the ET group, and VA supplementation could not inhibit this effect. These results may be explained by the differential effects of ET and VA on ROS production in different cell compartments. The effect of VA supplementation on antioxidant enzyme activity supports this hypothesis. 
The levels of both the antioxidant enzymes, SOD and CAT, were increased in the ET group, which is in accordance with the positive adaptations that occur as a consequence of ET. SOD and CAT activities are stimulated by increased substrate availability. Hence, when superoxide and hydrogen peroxide production is stimulated, their activities are increased. SOD is present in the mitochondria and the cytosol, and its activity is stimulated by superoxide production resulting from an increased demand for mitochondrial activity during intense exercise (Finaud et al. 2006; Myburgh 2014). The effects of chronic ET in the liver includes increased SOD activity and reduced lipid damage (da Silva et al. 2009), improved activity of the antioxidant enzyme glutathione peroxidase (Barcelos et al. 2014), and increased mitochondrial biogenesis and citrate synthase activity (Santos-Alves et al. 2015), all of which contribute to adaptation to increased ROS production and elevated aerobic demand for ATP synthesis. Increased reactive species production and redox imbalance result in upregulation of tissue defense, in order to cope with the adverse conditions (Banerjee et al. 2003). Supplementation with VA blocked the activation of SOD and CAT caused by ET, indicating that VA inhibited reactive species production, and thus SOD and CAT activities decreased due to reduced substrate availability. VA ( $\beta$-carotene) has previously been shown to have scavenging activity on several ROS, such as superoxide and peroxyl radicals (Yu 1994), which could have caused the decreased SOD activity in the ET+VA group. However, increased lipid damage and nitrotyrosine levels in the ET+VA group indicate that VA modulates SOD and CAT activity, but does not inhibit reactive species production.

ET is often associated with the prevention and treatment of lifestyle-related diseases (Neufer et al. 2015). This includes liver diseases; fat accumulation in the liver can lead to non-alcoholic fatty liver disease, and previous studies have reported that chronic aerobic exercise reduces this effect (Batatinha et al. 2016). Inflammation plays a 
key role in the development of this adverse liver condition (Nov et al. 2013). After intense ET, the levels of inflammatory cytokines rise significantly, and this response is often related to reactive species overload (Kosmidou et al. 2002). This effect may be followed by a compensatory response to increased production of anti-inflammatory mediators and activation of antioxidant enzymes. Here, we observed that VA supplementation combined with ET reduced the tissue levels of the pro-inflammatory cytokines, TNF- $\alpha$ and IL-1 $\beta$. It has been shown that ET does not affect the TNF- $\alpha$ expression levels in the liver, which is consistent with our results (E et al. 2013). One transcription factor that is associated with oxidative stress and inflammation is NF- $\mathrm{BB}$ (nuclear factor kappa-B), and it is also responsible for the regulation of TNF- $\alpha$ and IL$1 \beta$ expression (Barnes and Karin 1997). It has already been described that retinoic acid, the most active metabolic form of VA, can disrupt the nuclear translocation of NF- $\mathrm{kB}$ under inflammatory situations (Wang et al. 2015). Indeed, the NF- $\kappa \mathrm{B}$ pathway is among the most relevant signaling pathways in liver inflammation (He and Karin 2011).

Therefore, VA supplementation may inhibit the activation of $\mathrm{NF}-\kappa \mathrm{B}$, resulting in blunted pro-inflammatory cytokine release in response to intense exercise. Furthermore, the increase in IL-1 $\beta$ and IL-6 levels caused by ET or VA alone were inhibited when combined together. Similar effects have been observed in rats supplemented with caffeine and subjected to swimming ET (Cechella et al. 2014). IL-6 is considered a myokine and promotes anti-inflammatory actions in the muscle, as opposed to its proinflammatory actions in the liver and other tissues (Ost et al. 2016). In muscle cells, IL6 is responsible for increasing fat oxidation and stimulating glucose uptake by insulin; besides, it has been shown that IL- 6 deficient mice have reduced capacity to regenerate their liver (El-Kadre and Tinoco 2013). It is possible that ET enhances IL-6 levels in the liver as well as other tissues, where it promotes the inflammatory responses. 
Furthermore, the effect of ET on IL-10, a very effective anti-inflammatory cytokine, indicates that the pro-inflammatory effect of exercise on the liver is accompanied by the activation of anti-inflammatory response, and in combination with VA supplementation, this effect is further enhanced. We also evaluated the levels of RAGE, a multi-ligand receptor associated with inflammation in chronic diseases (Bohlooli et al. 2014; Schmidt 2015). Interestingly, SE+VA group showed enhanced RAGE levels, but both the ET and ET+VA groups showed decreased levels of RAGE. This is in agreement with the decrease in the levels of carboxymethyl-lysine, a key AGE that arises from non-enzymatic oxidative reactions between carbohydrates and proteins (Gaens et al. 2014), observed in all groups, as the regulation of this receptor depends on ligand availability. Thus, considering these effects, ET combined with VA supplementation induces an overall positive effect on pro-inflammatory activation induced by ET. It is important to evaluate several parameters when studying oxidative stress and inflammation, and interpreting one isolated parameter may lead to different conclusions. For example, VA is known to increase the production of superoxide anion (Murata and Kawanishi 2000), which could be responsible for the lower CAT activity observed in the ET+VA group, since high concentrations of superoxide can lead to CAT inhibition (Kono and Fridovich 1982; Pasquali et al. 2009). Also, oxidative stress and inflammation relationship goes both ways. During the inflammatory process phagocytes produce a large amount of ROS, for the purpose of eliminating the pathogen (Fialkow et al. 2007). On the other hand, high levels of hydrogen peroxide, ROS derivative from the dismutation of superoxide anion, can induce inflammation through NF- $\kappa$ B activation (Barnes and Karin 1997). Redox parameters alone can mislead the interpretation of results, hence the importance of evaluating, in this case, inflammatory parameters related to ET response. 
It is known that physical exercise increases the expression of proteins from the heat-shock family (Qu et al. 2015). Such a response generally protects cells from stress conditions caused by ET, such as redox imbalance, elevated body temperature, hypoxia, and glucose depletion (Krause et al. 2015). In the liver, a single bout of exercise is capable of enhancing the synthesis of HSP70 family proteins by 2-fold (Gonzalez and Manso 2004). Our ET protocol significantly increased liver HSP70 expression. A number of liver diseases show impaired HSP70 expression due to a decrease in heatshock transcription factor-1 (Qu et al. 2015), suggesting that ET may contribute to restoring basal levels of this protein. VA supplementation hindered the effect of ET on HSP70 levels, which may explain why the increase in nitrotyrosine by ET was not inhibited by VA supplementation, as HSP70 normally acts to prevent protein damage (Banerjee et al. 2003). Besides, as VA supplementation blocks the SOD and CAT activation caused by ET, the antioxidant response against ROS is impaired, which is in agreement with the increase in lipid damage and nitrotyrosine formation. In this context, despite the protective effect of inflammatory mediators, VA supplementation seems to impair antioxidant defense in the liver, thus contributing to the increase in oxidative lipid and protein damage during exercise.

The dose of choice for VA treatment in this study was based on the daily recommended value of $800 \mathrm{RE}$ for human adults (Institute of Medicine Panel on Micronutrients 2001) applying the HED, using the dose-by-factor approach (Nair and Jacob 2016). The food provided to the animals already contains VA at a dose that fulfills their daily recommendation; therefore, the combination with daily gavage supplementation is very likely to extrapolate the daily requirements for this vitamin. VA influences several metabolic processes (Chapman 2012), and its deficiency or excess may show very different effects. As described in the literature, higher doses would 
probably increase tissue damage (Pasquali et al. 2009; Gasparotto et al. 2015; Schnorr et al. 2015; Petiz et al. 2017), and lower doses may show no effect at all. Both VA and ET are potentially pro-oxidant to tissues when applied in excess, and we wanted to avoid potential harmful effects by using higher doses of this vitamin. Nonetheless, the dose of $450 \mathrm{RE}$ is below the tolerable VA daily upper intake level of $3000 \mathrm{RE}$, and the effects observed here could be considered mild.

\section{Conclusion}

VA, a redox-active molecule with known antioxidant properties, caused oxidative damage in the liver of trained rats. It increased lipid peroxidation and blunted the increased activity of antioxidant enzymes SOD and CAT caused by exercise alone. Even with this outcome, pro-inflammatory mediators (IL-1 $\beta$ and TNF- $\alpha$ ) decreased and anti-inflammatory (IL-10) increased in the group of trained rats supplemented with VA. This shows VA acting in an anti-inflammatory direction, regardless of the oxidative damage observed. Considering these effects, the dose of $450 \mathrm{RE}$ did not appear to cause liver injury, and suggests that controlled administration of VA for some types of exercise may imply anti-inflammatory properties. This was a low dosage of VA intake, and higher doses would probably increase the oxidative damage observed and modify the inflammatory response. Knowing that both VA and exercise are redox-modulators, it is important to conduct more studies with higher doses of VA and long-term exercised interventions, to determinate if the VA supplementation combined with exercise cause positive or negative effects. 


\section{Acknowledgements}

This work was supported by the Conselho Nacional de Desenvolvimento Científico e Tecnológico (CNPq) \#400437/2013-9, \#443514/2014-3 and \#401260/20143; Fundação de Amparo à Pesquisa do Estado do Rio Grande do Sul (FAPERGS) \#2299-2551/14-6; Pró-Reitoria de Pesquisa Universidade Federal do Rio Grande do Sul (Propesq-UFRGS) and Coordenação de Aperfeiçoamento de Pessoal de Nível Superior (CAPES). The funding agencies were not involved in the study design; collection, analysis, and interpretation of the data; in the writing of the report; and in the decision to submit the article for publication.

\section{Conflict of Interest statement}

The authors declare no conflict of interest regarding this study.

\section{References}

Abruzzo, P.M., Esposito, F., Marchionni, C., di Tullio, S., Belia, S., Fulle, S.,

Veicsteinas, A., Marini, M. 2013. Moderate exercise training induces ROS-related adaptations to skeletal muscles. Int. J. Sports. Med. 34(8): 676-87. doi:10.1055/s-00321323782

Aebi, H. 1984. Catalase in vitro. Methods. Enzymol. 105: 121-6.

Banerjee, A.K., Mandal, A., Chanda, D., Chakraborti, S. 2003. Oxidant, antioxidant and physical exercise. Mol. Cell. Biochem. 253(1-2): 307-12.

Banfi, G., Colombini, A., Lombardi, G., Lubkowska, A. 2012. Metabolic markers in sports medicine. Adv. Clin. Chem. 56: 1-54. 
Barcelos, R.P., Souza, M.A., Amaral, G.P., Stefanello, S.T., Bresciani, G., Fighera, M.R., Soares, F.A., Barbosa, N.V. 2014. Caffeine supplementation modulates oxidative stress markers in the liver of trained rats. Life. Sci. 96(1-2): 40-5.

doi:10.1016/j.1fs.2013.12.002

Barnes, P.J., Karin, M. 1997. Nuclear factor-kappaB: a pivotal transcription factor in chronic inflammatory diseases. N. Engl. J. Med. 336(15): 1066-71.

doi:10.1056/nejm199704103361506

Batatinha, H.A., Lima, E.A., Teixeira, A.A., Souza, C.O., Biondo, L.A., Silveira, L.S., Lira, F.S., Neto, J.C. 2016. Association between aerobic exercise and rosiglitazone avoided the NAFLD and liver inflammation exacerbated in PPAR-alpha knockout mice.

J. Cell. Physiol. doi:10.1002/jcp.25440

Blomhoff, R., Blomhoff, H.K. 2006. Overview of retinoid metabolism and function. J. Neurobiol. 66(7): 606-30. doi:10.1002/neu.20242

Bohlooli, M., Moosavi-Movahedi, A.A., Taghavi, F., Saboury, A.A., Maghami, P., Seyedarabi, A., Moosavi-Movahedi, F., Ahmad, F., Shockravi, A., Habibi-Rezaei, M. 2014. Inhibition of fluorescent advanced glycation end products (AGEs) of human serum albumin upon incubation with 3-beta-hydroxybutyrate. Mol. Biol. Rep. 41(6): 3705-13. doi:10.1007/s11033-014-3235-1

Brooks, G.A. 1986. The lactate shuttle during exercise and recovery. Med. Sci. Sports. Exerc. 18(3): 360-8.

Cechella, J.L., Leite, M.R., Dobrachinski, F., da Rocha, J.T., Carvalho, N.R., Duarte, M.M., Soares, F.A., Bresciani, G., Royes, L.F., Zeni, G. 2014. Moderate swimming exercise and caffeine supplementation reduce the levels of inflammatory cytokines without causing oxidative stress in tissues of middle-aged rats. Amino Acids, 46(5): 1187-95. doi:10.1007/s00726-014-1679-1 
Chance, B., Sies, H., Boveris, A. 1979. Hydroperoxide metabolism in mammalian organs. Physiol. Rev. 59(3): 527-605.

Chapman, M.S. 2012. Vitamin a: history, current uses, and controversies. Semin. Cutan. Med. Surg. 31(1): 11-6. doi:10.1016/j.sder.2011.11.009

Coker, R.H., Kjaer, M. 2005. Glucoregulation during exercise : the role of the neuroendocrine system. Sports Med. 35(7): 575-83.

D'Ambrosio, D.N., Clugston, R.D., Blaner, W.S. 2011. Vitamin A metabolism: an update. Nutrients, 3(1): 63-103. doi:10.3390/nu3010063

da Silva, L.A., Pinho, C.A., Rocha, L.G., Tuon, T., Silveira, P.C., Pinho, R.A. 2009. Effect of different models of physical exercise on oxidative stress markers in mouse liver. Appl. Physiol. Nutr. Metab. 34(1): 60-5. doi:10.1139/h08-132 de Oliveira, M.R., Oliveira, M.W., Lorenzi, R., Fagundes da Rocha, R., Fonseca Moreira, J.C. 2009. Short-term vitamin A supplementation at therapeutic doses induces a pro-oxidative state in the hepatic environment and facilitates calcium-ion-induced oxidative stress in rat liver mitochondria independently from permeability transition pore formation : detrimental effects of vitamin A supplementation on rat liver redox and bioenergetic states homeostasis. Cell. Biol. Toxicol. 25(6): 545-60. doi:10.1007/s10565$008-9111-9$

Draper, H.H., Hadley, M. 1990. Malondialdehyde determination as index of lipid peroxidation. Methods Enzymol. 186: 421-31.

E, L., Lu, J., Burns, J.M., Swerdlow, R.H. 2013. Effect of exercise on mouse liver and brain bioenergetic infrastructures. Exp. Physiol. 98(1): 207-19.

doi:10.1113/expphysiol.2012.066688 
El-Kadre, L.J., Tinoco, A.C. 2013. Interleukin-6 and obesity: the crosstalk between intestine, pancreas and liver. Curr. Opin. Clin. Nutr. Metab. Care, 16(5): 564-8. doi:10.1097/MCO.0b013e32836410e6

Fialkow, L., Wang, Y., Downey, G.P. 2007. Reactive oxygen and nitrogen species as signaling molecules regulating neutrophil function. Free Radic. Biol. Med. 42(2): 15364. doi:10.1016/j.freeradbiomed.2006.09.030

Finaud, J., Lac, G., Filaire, E. 2006. Oxidative stress : relationship with exercise and training. Sports Med. 36(4): 327-58.

Gaens, K.H., Goossens, G.H., Niessen, P.M., van Greevenbroek, M.M., van der Kallen, C.J., Niessen, H.W., Rensen, S.S., Buurman, W.A., Greve, J.W., Blaak, E.E., van Zandvoort, M.A., Bierhaus, A., Stehouwer, C.D., Schalkwijk, C.G. 2014. Nepsilon(carboxymethyl)lysine-receptor for advanced glycation end product axis is a key modulator of obesity-induced dysregulation of adipokine expression and insulin resistance. Arterioscler. Thromb. Vasc. Biol. 34(6): 1199-208.

doi:10.1161/atvbaha.113.302281

Garcia-Cortes, M., Robles-Diaz, M., Ortega-Alonso, A., Medina-Caliz, I., Andrade, R.J. 2016. Hepatotoxicity by Dietary Supplements: A Tabular Listing and Clinical Characteristics. Int. J. Mol. Sci. 17(4): 537. doi:10.3390/ijms17040537

Gasparotto, J., Petiz, L.L., Girardi, C.S., Bortolin, R.C., de Vargas, A.R., Henkin, B.S., Chaves, P.R., Roncato, S., Matte, C., Zanotto-Filho, A., Moreira, J.C., Gelain, D.P. 2015. Supplementation with vitamin A enhances oxidative stress in the lungs of rats submitted to aerobic exercise. Appl. Physiol. Nutr. Metab. 40(12): 1253-61.

doi:10.1139/apnm-2015-0218 
Geubel, A.P., De Galocsy, C., Alves, N., Rahier, J., Dive, C. 1991. Liver damage caused by therapeutic vitamin A administration: estimate of dose-related toxicity in 41 cases. Gastroenterology, 100(6): 1701-9.

Gleeson, M. 2007. Immune function in sport and exercise. J. Appl. Physiol. (1985) 103(2): 693-9. doi:10.1152/japplphysiol.00008.2007

Gleeson, M., Bishop, N.C., Stensel, D.J., Lindley, M.R., Mastana, S.S., Nimmo, M.A. 2011. The anti-inflammatory effects of exercise: mechanisms and implications for the prevention and treatment of disease. Nat. Rev. Immunol. 11(9): 607-15.

doi:10.1038/nri3041

Gobatto, C.A., de Mello, M.A., Sibuya, C.Y., de Azevedo, J.R., dos Santos, L.A., Kokubun, E. 2001. Maximal lactate steady state in rats submitted to swimming exercise. Comp. Biochem. Physiol. A. Mol. Integr. Physiol. 130(1): 21-7.

Gonzalez, B., Manso, R. 2004. Induction, modification and accumulation of HSP70s in the rat liver after acute exercise: early and late responses. J. Physiol. 556(Pt 2): 369-85. doi:10.1113/jphysiol.2003.058420

Halliwell, B. 2006. Reactive Species and Antioxidants. Redox Biology Is a Fundamental Theme of Aerobic Life. Plant Physiology, 141(2): 312-322. doi:10.1104/pp.106.077073

He, G., Karin, M. 2011. NF-kappaB and STAT3 - key players in liver inflammation and cancer. Cell Res. 21(1): 159-68. doi:10.1038/cr.2010.183

Institute of Medicine Panel on Micronutrients. 2001. Dietary Reference Intakes for Vitamin A, Vitamin K, Arsenic, Boron, Chromium, Copper, Iodine, Iron, Manganese, Molybdenum, Nickel, Silicon, Vanadium, and Zinc. Washington (DC): National Academies Press, US. 
Jones, D.P. 2006. Redefining oxidative stress. Antioxid. Redox. Signal. 8(9-10): 186579. doi:10.1089/ars.2006.8.1865

Kayatekin, B.M., Gonenc, S., Acikgoz, O., Uysal, N., Dayi, A. 2002. Effects of sprint exercise on oxidative stress in skeletal muscle and liver. Eur. J. Appl. Physiol. 87(2): 141-4. doi:10.1007/s00421-002-0607-3

Kono, Y., Fridovich, I. 1982. Superoxide radical inhibits catalase. J. Biol. Chem. 257(10): 5751-4.

Kosmidou, I., Vassilakopoulos, T., Xagorari, A., Zakynthinos, S., Papapetropoulos, A., Roussos, C. 2002. Production of interleukin-6 by skeletal myotubes: role of reactive oxygen species. Am. J. Respir. Cell Mol. Biol. 26(5): 587-93.

doi:10.1165/ajrcmb.26.5.4598

Koury, J.C., Daleprane, J.B., Pitaluga-Filho, M.V., de Oliveira, C.F., Goncalves, M.C., Passos, M.C. 2016. Aerobic Conditioning Might Protect Against Liver and Muscle Injury Caused by Short-Term Military Training. J. Strength Cond. Res. 30(2): 454-60. doi:10.1519/jsc.0000000000001102

Krause, M., Heck, T.G., Bittencourt, A., Scomazzon, S.P., Newsholme, P., Curi, R., Homem de Bittencourt, P.I., Jr. 2015. The chaperone balance hypothesis: the importance of the extracellular to intracellular HSP70 ratio to inflammation-driven type 2 diabetes, the effect of exercise, and the implications for clinical management. Mediators Inflamm. 2015: 249205. doi:10.1155/2015/249205

Kunzler, A., Zeidan-Chulia, F., Gasparotto, J., Girardi, C.S., Klafke, K., Petiz, L.L., Bortolin, R.C., Rostirolla, D.C., Zanotto-Filho, A., de Bittencourt Pasquali, M.A., Dickson, P., Dunkley, P., Moreira, J.C., Gelain, D.P. 2016. Changes in Cell Cycle and Up-Regulation of Neuronal Markers During SH-SY5Y Neurodifferentiation by Retinoic 
Acid are Mediated by Reactive Species Production and Oxidative Stress. Mol.

Neurobiol. doi:10.1007/s12035-016-0189-4

Levine, R.L., Garland, D., Oliver, C.N., Amici, A., Climent, I., Lenz, A.G., Ahn, B.W., Shaltiel, S., Stadtman, E.R. 1990. Determination of carbonyl content in oxidatively modified proteins. Methods Enzymol. 186: 464-78.

Lima, T.I., Monteiro, I.C., Valenca, S., Leal-Cardoso, J.H., Fortunato, R.S., Carvalho, D.P., Teodoro, B.G., Ceccatto, V.M. 2015. Effect of exercise training on liver antioxidant enzymes in STZ-diabetic rats. Life Sci. 128: 64-71.

doi:10.1016/j.1fs.2015.01.031

Lowry, O.H., Rosebrough, N.J., Farr, A.L., Randall, R.J. 1951. Protein measurement with the Folin phenol reagent. J. Biol. Chem. 193(1): 265-75.

Misra, H.P., Fridovich, I. 1972. The role of superoxide anion in the autoxidation of epinephrine and a simple assay for superoxide dismutase. J. Biol. Chem. 247(10): 31705.

Murata, M., Kawanishi, S. 2000. Oxidative DNA damage by vitamin A and its derivative via superoxide generation. J. Biol. Chem. 275(3): 2003-8.

Myburgh, K.H. 2014. Polyphenol supplementation: benefits for exercise performance or oxidative stress? Sports Med. 44 Suppl 1: S57-70. doi:10.1007/s40279-014-0151-4 Nair, A.B., Jacob, S. 2016. A simple practice guide for dose conversion between animals and human. J. Basic Clin. Pharm. 7(2): 27-31. doi:10.4103/0976-0105.177703 Neufer, P.D., Bamman, M.M., Muoio, D.M., Bouchard, C., Cooper, D.M., Goodpaster, B.H., Booth, F.W., Kohrt, W.M., Gerszten, R.E., Mattson, M.P., Hepple, R.T., Kraus, W.E., Reid, M.B., Bodine, S.C., Jakicic, J.M., Fleg, J.L., Williams, J.P., Joseph, L., Evans, M., Maruvada, P., Rodgers, M., Roary, M., Boyce, A.T., Drugan, J.K., Koenig, J.I., Ingraham, R.H., Krotoski, D., Garcia-Cazarin, M., McGowan, J.A., Laughlin, M.R. 
2015. Understanding the Cellular and Molecular Mechanisms of Physical ActivityInduced Health Benefits. Cell Metab. 22(1): 4-11. doi:10.1016/j.cmet.2015.05.011 Nov, O., Shapiro, H., Ovadia, H., Tarnovscki, T., Dvir, I., Shemesh, E., Kovsan, J., Shelef, I., Carmi, Y., Voronov, E., Apte, R.N., Lewis, E., Haim, Y., Konrad, D., Bashan, N., Rudich, A. 2013. Interleukin-1beta regulates fat-liver crosstalk in obesity by auto-paracrine modulation of adipose tissue inflammation and expandability. PLoS One, 8(1): e53626. doi:10.1371/journal.pone.0053626

Oh, S., Tanaka, K., Tsujimoto, T., So, R., Shida, T., Shoda, J. 2014. Regular exercise coupled to diet regimen accelerates reduction of hepatic steatosis and associated pathological conditions in nonalcoholic fatty liver disease. Metab. Syndr. Relat. Disord. 12(5): 290-8. doi:10.1089/met.2013.0143

Ordonez, R., Carbajo-Pescador, S., Mauriz, J.L., Gonzalez-Gallego, J. 2015. Understanding nutritional interventions and physical exercise in non-alcoholic fatty liver disease. Curr. Mol. Med. 15(1): 3-26.

Ost, M., Coleman, V., Kasch, J., Klaus, S. 2016. Regulation of myokine expression: Role of exercise and cellular stress. Free Radic. Biol. Med. 98: 78-89. doi:10.1016/j.freeradbiomed.2016.02.018 Ozhogina, O.A., Kasaikina, O.T. 1995. Beta-carotene as an interceptor of free radicals. Free Radic. Biol. Med. 19(5): 575-81.

Pasquali, M.A., Gelain, D.P., Oliveira, M.R., Behr, G.A., Motta, L.L., Rocha, R.F., Klamt, F., Moreira, J.C. 2009. Vitamin A supplementation induces oxidative stress and decreases the immunocontent of catalase and superoxide dismutase in rat lungs. Exp. Lung Res. 35(5): 427-38.

Petiz, L.L., Girardi, C.S., Bortolin, R.C., Kunzler, A., Gasparotto, J., Rabelo, T.K., Matte, C., Moreira, J.C., Gelain, D.P. 2017. Vitamin A Oral Supplementation Induces 
Oxidative Stress and Suppresses IL-10 and HSP70 in Skeletal Muscle of Trained Rats. Nutrients, 9(4). doi:10.3390/nu9040353

Pillon Barcelos, R., Freire Royes, L.F., Gonzalez-Gallego, J., Bresciani, G. 2017.

Oxidative stress and inflammation: liver responses and adaptations to acute and regular exercise. Free Radic. Res. 51(2): 222-236. doi:10.1080/10715762.2017.1291942

Powers, S.K., Lennon, S.L. 1999. Analysis of cellular responses to free radicals: focus on exercise and skeletal muscle. Proc. Nutr. Soc. 58(4): 1025-33.

Qu, B., Jia, Y., Liu, Y., Wang, H., Ren, G., Wang, H. 2015. The detection and role of heat shock protein 70 in various nondisease conditions and disease conditions: a literature review. Cell Stress Chaperones, 20(6): 885-92. doi:10.1007/s12192-015-06188

Radak, Z., Zhao, Z., Koltai, E., Ohno, H., Atalay, M. 2013. Oxygen consumption and usage during physical exercise: the balance between oxidative stress and ROSdependent adaptive signaling. Antioxid. Redox Signal. 18(10): 1208-46. doi:10.1089/ars.2011.4498

Santos-Alves, E., Marques-Aleixo, I., Rizo-Roca, D., Torrella, J.R., Oliveira, P.J., Magalhaes, J., Ascensao, A. 2015. Exercise modulates liver cellular and mitochondrial proteins related to quality control signaling. Life Sci. 135: 124-30.

doi:10.1016/j.1fs.2015.06.007

Sarsour, E.H., Kumar, M.G., Chaudhuri, L., Kalen, A.L., Goswami, P.C. 2009. Redox control of the cell cycle in health and disease. Antioxid. Redox. Signal. 11(12): 29853011. doi:10.1089/ars.2009.2513

Schmidt, A.M. 2015. Soluble RAGEs - Prospects for treating \& tracking metabolic and inflammatory disease. Vascul. Pharmacol. 72: 1-8. doi:10.1016/j.vph.2015.06.011 
Schnorr, C.E., Bittencourt Lda, S., Petiz, L.L., Gelain, D.P., Zeidan-Chulia, F., Moreira, J.C. 2015. Chronic retinyl palmitate supplementation to middle-aged Wistar rats disrupts the brain redox homeostasis and induces changes in emotional behavior. Mol. Nutr. Food Res. 59(5): 979-90. doi:10.1002/mnfr.201400637

Schnorr, C.E., Morrone Mda, S., Weber, M.H., Lorenzi, R., Behr, G.A., Moreira, J.C. 2011. The effects of vitamin A supplementation to rats during gestation and lactation upon redox parameters: increased oxidative stress and redox modulation in mothers and their offspring. Food Chem. Toxicol. 49(10): 2645-54. doi:10.1016/j.fct.2011.07.010 Schroder, H., Navarro, E., Mora, J., Galiano, D., Tramullas, A. 2001. Effects of alphatocopherol, beta-carotene and ascorbic acid on oxidative, hormonal and enzymatic exercise stress markers in habitual training activity of professional basketball players. Eur. J. Nutr. 40(4): 178-84.

Sen, C.K. 1995. Oxidants and antioxidants in exercise. J. Appl. Physiol. (1985) 79(3): 675-86.

Shephard, R.J., Johnson, N. 2015. Effects of physical activity upon the liver. Eur. J. Appl. Physiol. 115(1): 1-46. doi:10.1007/s00421-014-3031-6

Shimizu, H., Tsubota, T., Kanki, K., Shiota, G. 2017. All-trans retinoic acid ameliorates hepatic stellate cell activation via suppression of thioredoxin interacting protein expression. J. Cell Physiol. doi:10.1002/jcp.25921

Wang, G., Xiu, P., Li, F., Xin, C., Li, K. 2014. Vitamin A supplementation alleviates extrahepatic cholestasis liver injury through Nrf2 activation. Oxid. Med. Cell Longev. 2014: 273692. doi:10.1155/2014/273692

Wang, R., Chen, S., Liu, Y., Diao, S., Xue, Y., You, X., Park, E.A., Liao, F.F. 2015. All-trans-retinoic acid reduces BACE1 expression under inflammatory conditions via 
modulation of nuclear factor kappaB (NFkappaB) signaling. J. Biol. Chem. 290(37): 22532-42. doi:10.1074/jbc.M115.662908

Wautier, M.P., Guillausseau, P.J., Wautier, J.L. 2016. Activation of the receptor for advanced glycation end products and consequences on health. Diabetes Metab. Syndr. doi:10.1016/j.dsx.2016.09.009

Yu, B.P. 1994. Cellular defenses against damage from reactive oxygen species. Physiol. Rev. 74(1): 139-62.

\section{Captions for figures}

Fig 1. Effects of exercise training and vitamin A supplementation on serum levels of hepatic enzymes ALT (A) and AST (B). Data presented as main \pm SEM (n=6-8). * $\mathrm{p}<0.05$ significant difference from SE group. ${ }^{\mathrm{a}} \mathrm{p}<0.05$ significant difference from SE+VA group using one-way ANOVA followed by Bonferroni's posthoc test.

Fig 2. Effects of exercise training and vitamin A supplementation on liver oxidative stress markers and stress parameters. TBARS (A), 4-HNE (B), carbonyl (C), nitrotyrosine (D), carboxymethyl lysine (E), HSP70 (F). Data presented as main \pm SEM (n=6-8). $* \mathrm{p}<0.05 * * * \mathrm{p}<0.001$ significant difference from SE group. ${ }^{\text {aa }} \mathrm{p}<0.01$ aaa $\mathrm{p}<0.001$ significant difference from $\mathrm{SE}+\mathrm{VA}$ group. ${ }^{\# \#} \mathrm{p}<0.01{ }^{\# \#} \mathrm{p}<0.001$ significant difference from ET group using one-way ANOVA followed by Bonferroni's posthoc test. 
Fig 3. Effects of exercise training and vitamin A supplementation on the activity of liver antioxidant enzymes SOD (A) and CAT (B). Data presented as main \pm SEM ( $\mathrm{n}=6-8)$ * $\mathrm{p}<0.05$ significant difference from SE group. ${ }^{\#} \mathrm{p}<0.05^{\# \#} \mathrm{p}<0.001$ significant difference from ET group using one-way ANOVA followed by Bonferroni's posthoc test.

Fig 4. Effects of exercise training and vitamin A supplementation on liver inflammation parameters. IL-1 $\beta$ (A), TNF- $\alpha$ (B), IL-6 (C), IL-10 (D), RAGE (E). Data presented as main $\pm \operatorname{SEM}(\mathrm{n}=6-8) .{ }^{*} \mathrm{p}<0.05 * * \mathrm{p}<0.01 * * * \mathrm{p}<0.001$ significant difference from SE group. aaa $\mathrm{p}<0.001$ significant difference from $\mathrm{SE}+\mathrm{VA}$ group. ${ }^{\# \#} \mathrm{p}<0.001$ significant difference from ET group using one-way ANOVA followed by Bonferroni's posthoc test. 

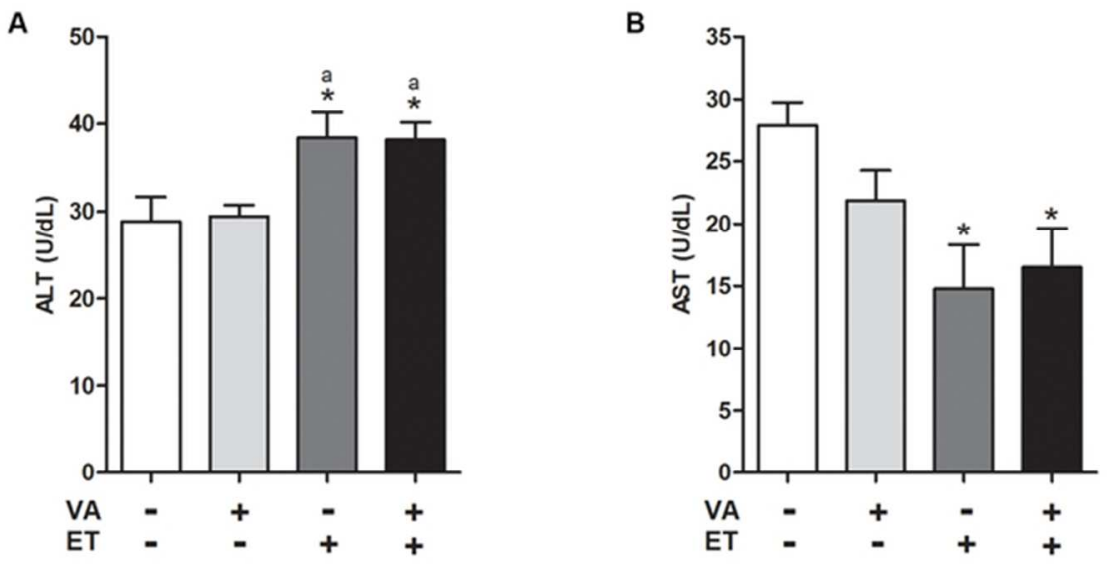

Fig 1. Effects of exercise training and vitamin A supplementation on serum levels of hepatic enzymes ALT $(A)$ and AST (B). Data presented as main \pm SEM $(n=6-8)$. $* p<0.05$ significant difference from SE group. a $p<0.05$ significant difference from SE+VA group using one-way ANOVA followed by Bonferroni's posthoc test.

$59 \times 27 \mathrm{~mm}(300 \times 300 \mathrm{DPI})$ 
A
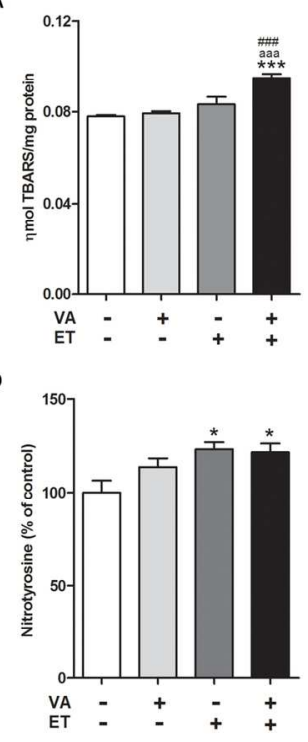

B

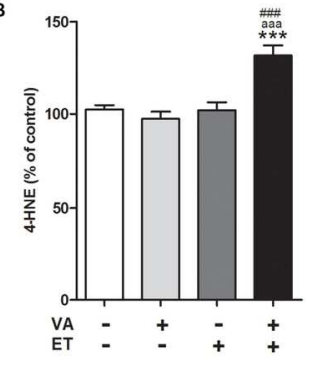

E

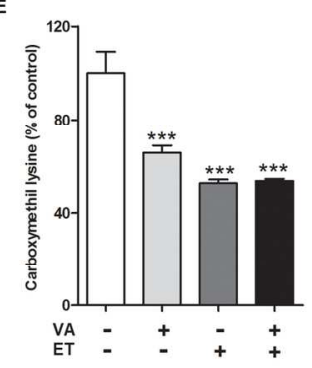

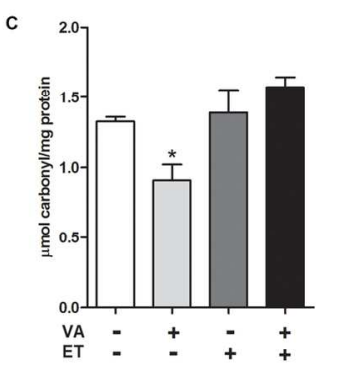

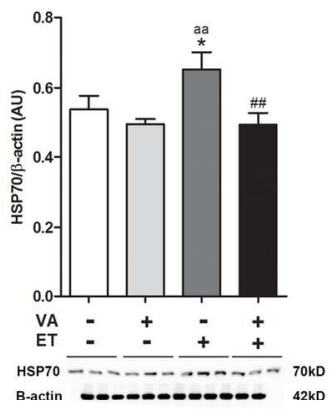

Fig 2. Effects of exercise training and vitamin A supplementation on liver oxidative stress markers and stress parameters. TBARS (A), 4-HNE (B), carbonyl (C), nitrotyrosine (D), carboxymethyl lysine (E), HSP70 (F). Data presented as main \pm SEM $(n=6-8)$. $* p<0.05 * * * p<0.001$ significant difference from SE group. aa $p<0.01$ aaa $p<0.001$ significant difference from SE+VA group. \#\# $p<0.01$ \#\#\# $p<0.001$ significant difference from ET group using one-way ANOVA followed by Bonferroni's posthoc test.

$149 \times 112 \mathrm{~mm}(300 \times 300 \mathrm{DPI})$ 
A

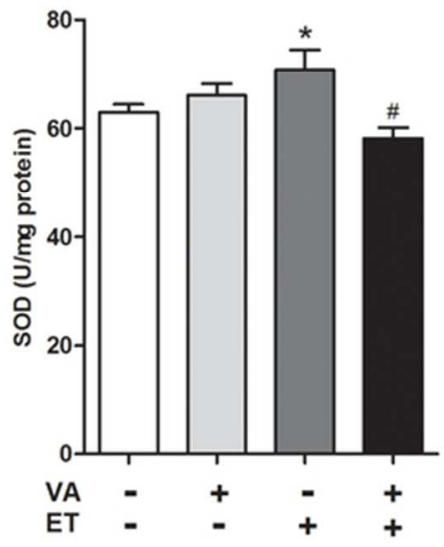

B

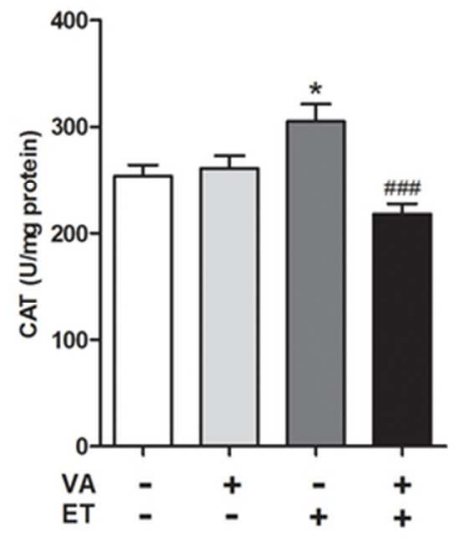

Fig 3. Effects of exercise training and vitamin A supplementation on the activity of liver antioxidant enzymes SOD $(A)$ and CAT (B). Data presented as main $\pm \operatorname{SEM}(n=6-8)$. $* p<0.05$ significant difference from SE group. \# $p<0.05$ \#\# $\mathrm{p}<0.001$ significant difference from ET group using one-way ANOVA followed by Bonferroni's posthoc test.

$59 \times 27 \mathrm{~mm}(300 \times 300 \mathrm{DPI})$ 
A

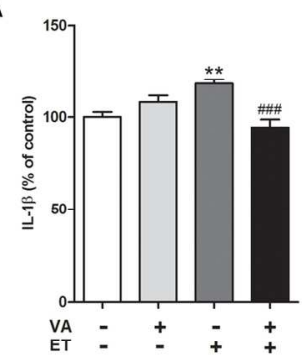

D

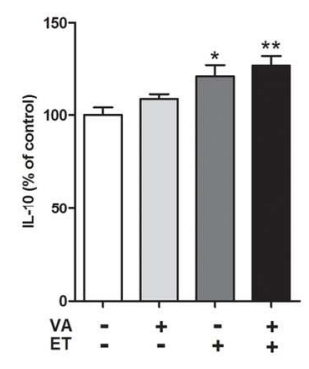

B

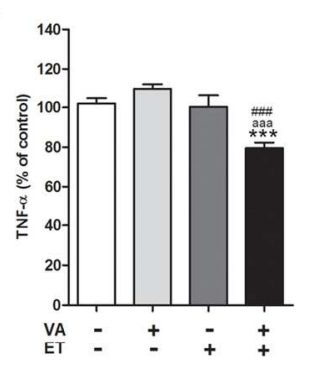

$\mathrm{E}$

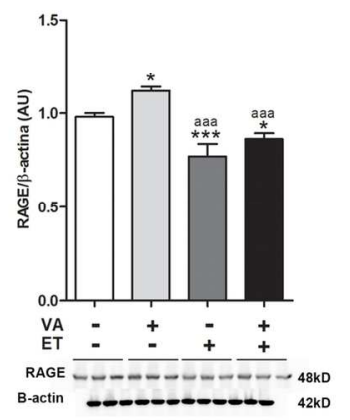

c

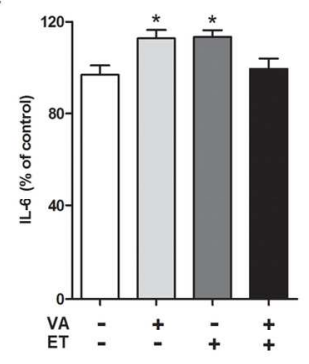

Fig 4. Effects of exercise training and vitamin A supplementation on liver inflammation parameters. IL-1 $\beta$ (A), TNF-a (B), IL-6 (C), IL-10 (D), RAGE (E). Data presented as main \pm SEM $(n=6-8) . * p<0.05 * * p<0.01$ $* * * p<0.001$ significant difference from SE group. aaa $\mathrm{p}<0.001$ significant difference from SE+VA group. \#\# \# $p<0.001$ significant difference from ET group using one-way ANOVA followed by Bonferroni's posthoc test.

$139 \times 97 \mathrm{~mm}(300 \times 300$ DPI $)$ 